\title{
Taxa respiratória de frutos de juazeiro (Ziziphus joazeiro Mart.) armazenado sob temperatura ambiente
}

\section{Respiratory rate of juazeiro fruits (Ziziphus joazeiro Mart.) stored under ambient temperature}

\author{
Jéssica Leite da Silva ${ }^{1 *}$, Franciscleudo Bezerra da Costa ${ }^{2}$, Ana Marinho do Nascimento ${ }^{3}$, Francimalba Francilda de Sousa ${ }^{4}$ \\ Kalinne Passos dos Santos 5
}

Resumo: O juazeiro é uma planta bastante conhecida dos sertanejos, que além de servir de sombra e arborização de ruas, seus frutos são amplamente consumidos por animais. Algumas pesquisas são direcionadas a essa cultura, no entanto, não existem pesquisas no que diz respeito à natureza respiratória dos frutos. Assim, esta pesquisa teve por objetivo avaliar a taxa respiratória de frutos de juazeiro colhidos de plantas localizadas no município de Queimadas, Paraíba, armazenados à temperatura ambiente controlada $\left(23 \pm 1{ }^{\circ} \mathrm{C}\right.$ e $55 \pm 2 \%$ UR) por quatro dias. A taxa respiratória foi determinada por meio da metodologia descrita por Crispim et al. (1994) com adaptações, também foi estimada a massa fresca do fruto e a perda de massa durante o período de armazenamento . Os resultados demonstraram redução de massa fresca do fruto, aumento de perda de massa, bem como, picos climatéricos no terceiro dia de armazenamento, podendo assim, serem considerados frutos de natureza climatérica.

Palavras-chave: Respiração; Armazenamento; Pico climatérico.

Abstract: The juazeiro is a well-known plant of the sertanejos, that besides serving of shade and arborization of streets, its fruits are widely consumed by animals. Some research is directed to this culture, however, there is no research regarding the respiratory nature of the fruits. The objective of this research was to evaluate the respiratory rate of juazeiro fruits harvested from plants located in the city of Queimadas, Paraíba, stored at controlled ambient temperature $\left(23 \pm 1^{\circ} \mathrm{C}\right.$ and $\left.55 \pm 2 \% \mathrm{RH}\right)$ for four days. The respiratory rate was determined using the methodology described by Crispim et al. (1994) with adaptations, it was also estimated the fresh mass of the fruit and the loss of mass during the storage period. The results showed a reduction of fresh mass of the fruit, increase of mass loss, as well as, climacteric peaks on the third day of storage, thus being considere fruits of climacteric nature.

Key words: Breath; storage; climacteric peak.

\footnotetext{
*Autor para correspondência

Recebido para publicação em 08/02/2017; aprovado em 17/05/2017

${ }^{1}$ Doutoranda em Engenharia de Processos, UFCG, Campus Campina Grande-PB, e-mail: jessicaleite2010@gmail.com

${ }^{2}$ Professor do Centro de Ciências e Tecnologia Agroalimentar, UFCG, Campus Pombal-PB, e-mail: franciscleudo@yahoo.com.br

${ }^{3}$ Doutoranda em Engenharia de Processos, UFCG, Campus Campina Grande-PB, e-mail: anamarinho06@hotmail.com

${ }^{4}$ Graduanda em Agronomia, UFCG, Campus Pombal-PB, e-mail: malbah_senna@ hotmail.com

${ }^{4}$ Graduanda em Engenharia de Alimentos, UFCG, Campus Pombal-PB, e-mail: kalinne2012.2@ gmail.com
} 


\section{INTRODUÇÃO}

O juazeiro (Ziziphus joazeiro Mart. Rhamnaceae) popularmente chamado de "juá" é uma espécie típica do Nordeste Brasileiro, que se apresenta de forma isolada nas matas xerófitas da Caatinga. É uma árvore bastante conhecida no semiárido nordestino, que apresenta uma grande importância econômica, especialmente devido as suas propriedades medicinais. Os frutos dessa planta apresentam características nutricionais podendo até ser utilizado para alimentação humana (DANTAS et al., 2014). Os sertanejos o consideram uma planta especial, porque, mesmo em épocas de seca prolongada, está sempre verde e com flores. Essa alta tolerância à seca é motivada pelas suas raízes que atingem grandes profundidades (BRAGA, 2010). É uma árvore que cresce lentamente e vive mais de 100 anos (LOPPES, 2008).

Possui grande importância econômica e ecológica, sendo utilizada localmente para produção de lenha e carvão, arborização de ruas e jardins, além de possuir frutos comestíveis, os quais são explorados de forma extrativista (LORENZI, 2009). É empregado na fabricação de sabão, dentifrício e na indústria madeireira (LORENZI; MATOS, 2008). Além disso, é uma das espécies do bioma caatinga bastante utilizada na medicina popular como expectorante, no tratamento de bronquites e de úlceras gástricas, na fabricação de cosméticos e creme dental, além de servir na alimentação de animais principalmente nos períodos de seca (LORENZI, 2009).

A sua frutificação ocorre de junho a julho em regiões semiáridas como as do Ceará, Pernambuco, Paraíba e outras. Os seus frutos quando maduros possuem uma drupa globosa de cor amarelada, com comprimento de 1,5 a $2 \mathrm{~cm}$ respectivamente. Eles fornecem uma polpa carnosa adocicada e ácida. Os mesmos possuem uma casca fina que recobre toda polpa farinácea, já no seu interior encontram-se as sementes que são envolvidas por uma mucilagem transparente (CARVALHO, 2007). Entre os constituintes químicos do juazeiro estão a saponina, os hidratos de carbono, a celulose e a vitamina $C$, sendo que essa última se apresenta em grande concentração em seus frutos (DANTAS et al., 2014).

A respiração é um processo envolvido na fisiologia póscolheita de frutos e hortaliças. Os acréscimos da temperatura em produtos de origem vegetal por muitas vezes fornecem um avanço da taxa de respiração. Essa ocorrência reduz a vida útil desses alimentos, isso porque esse parâmetro está relacionado com as consequências da ação da temperatura nos frutos. Durante a respiração várias reações metabólicas ocorrem devido a fatores como temperatura, transpiração e a concentração de gases como $\mathrm{CO}_{2}, \mathrm{O}_{2}$ e etileno. As concentrações reduzidas de $\mathrm{O}_{2}$ e elevadas de $\mathrm{CO}_{2}$ no período de armazenamento favorecem reduções de longo prazo na taxa de respiração, que consequentemente aumenta a vida útil dos produtos hortícolas após a colheita (CALBO et al., 2007).

A verificação da respiração é um atributo de extrema importância para o amadurecimento dos frutos. Informações sobre essa propriedade auxiliam na manutenção e capacidade de armazenamento desses produtos. Sabe-se que as lesões ocasionadas nos tecidos dos frutos induzem o avanço na taxa respiratória e quanto maior o nível dos cortes, mais elevada será a taxa de respiração, portanto, o conhecimento sobre os efeitos da respiração nos frutos é indispensável para manter a qualidade desses alimentos (FONSECA et al., 2002).
O presente trabalho teve como objetivo avaliar a taxa de respiração dos frutos de juazeiro colhidos no município de Queimadas localizado no agreste da Paraíba, submetidos ao armazenamento sob temperatura ambiente controlada.

\section{MATERIAL E MÉTODOS}

\section{Obtenção do material vegetal}

Foram utilizados frutos de juazeiro, oriundos de uma planta localizada no município de Queimadas no estado da Paraíba (Figura 1), colhidos em junho de 2016.

Figura 1. Planta de Zizyphus joazeiro Mart., onde foram colhidos os frutos utilizados no experimento, Pombal, Paraíba

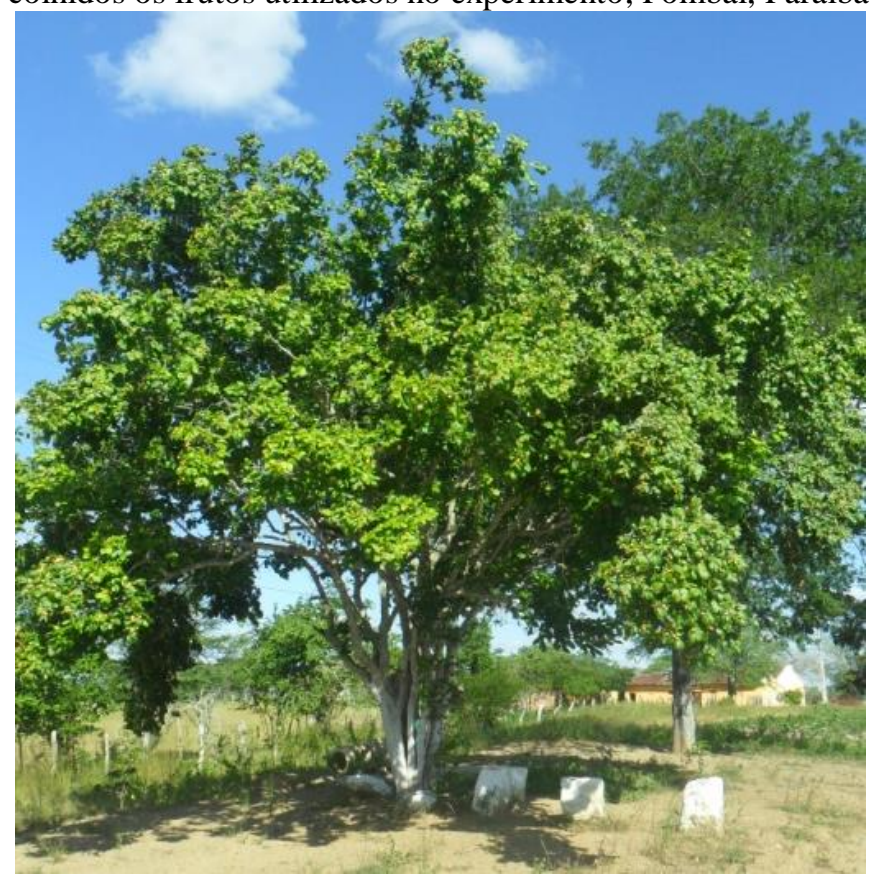

Fonte: Autores (2016)

\section{Procedimento experimental}

O procedimento experimental foi executado conforme fluxograma ilustrado na Figura 2.

Figura 2. Fluxograma de execução do procedimento experimental, Pombal, Paraíba

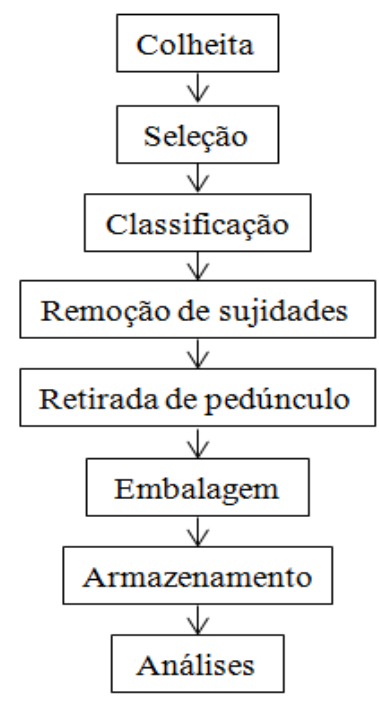

Fonte: Autores (2016) 
Os frutos foram colhidos manualmente no início da manhã, das 7:00 às 8:00 h, acondicionados em sacos de polietileno, e transportados em carro com temperatura controlada por meio de ar-condicionado ao laboratório de Química, Bioquímica e Análise de Alimentos do CCTA/Pombal.

Os frutos foram selecionados visualmente quanto à ausência de injúrias, de modo a obter amostras uniformes e com qualidade.

Os frutos empregados neste estudo foram os classificados como IV estádio de maturação, segundo classificação definida por Silva (2015). Os frutos deste estado de maturação apresentaram a casca totalmente amarela (Figura 3), sendo considerado totalmente maduro.

Figura 3. Frutos de juazeiro classificados no quarto estádio de maturação. Pombal, Paraíba

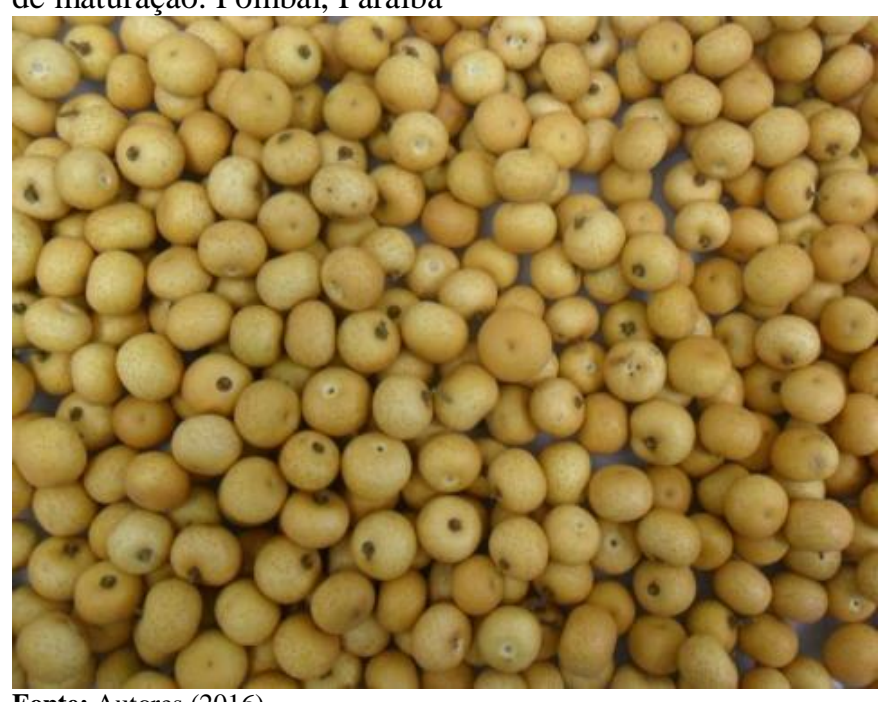

Fonte: Autores (2016).

A higienização não foi realizada em água corrente, nem sanitização, pois os frutos de juazeiro apresentam elevada taxa metabólica e o contato com água intensifica os processos de senescência, como foi relatado por Silva (2015), em que os estádios de maturação mais avançados (III, IV e V) não mostraram-se aptos à realização das análises durante o armazenamento proposto na pesquisa (6 dias). Foi realizada a remoção das sujidades aparentes com auxílio de papel toalha e remoção do pedúnculo dos frutos manualmente.

Os frutos foram embalados em bandejas de poliestireno expandido, envolvidas por filme de PVC $12 \mu \mathrm{m}$, cada bandeja continha aproximadamente $100 \mathrm{~g}$ de frutos de juazeiro.

As bandejas foram dispostas sob bancada e mantidas sob temperatura ambiente controlada por meio de ar condicionado $\left(23 \pm 1{ }^{\circ} \mathrm{C}\right.$ e $55 \pm 2 \%$ UR) por quadro dias.

Foram realizadas análises de massa fresca, perda de massa e taxa respiratória em todos os dias de armazenamento, configurando cinco tempos de análises $(0,1,2,3$ e 4 dias). Foram elaboradas cinco bandejas para cada dia de armazenamento.

A massa fresca ( $\mathrm{g}$ ) foi quantificada gravimetricamente a partir dos frutos inteiros em balança semianalítica com capacidade de $600 \mathrm{~g}$ e resolução $0,01 \mathrm{~g}$.

A análise de perda de massa foi calculada pela diferença entre a massa inicial e a obtida em cada intervalo de tempo, utilizando balança semi-analítica da marca Bel com capacidade de $600 \mathrm{~g}$ e resolução $0,01 \mathrm{~g}$.
A taxa respiratória (mg CO $2 . \mathrm{g}^{-1} \mathrm{MF}$ ) foi determinada de acordo com Crispim et al. (1994) com adaptações.

Os frutos foram acondicionados no interior de potes de polietileno com tampa, com capacidade para $0,15 \mathrm{~L}$, por 12 horas a cada dia de armazenamento e ao lado destes (frutos), dentro dos recipientes foi colocado outro recipiente contendo $\mathrm{NaOH}$ 0,5 mol. $\mathrm{L}^{-1}$, que funciona como fixador do $\mathrm{CO}_{2}$ produzido no processo de respiração. Para evitar trocas gasosas com o meio, as tampas dos recipientes foram envolvidas por película de silicone.

Após $12 \mathrm{~h}$ de inoculo, a solução de $\mathrm{NaOH}$ foi retirada do recipiente e recebeu três gotas do indicador fenolftaleína e 10 $\mathrm{mL}$ de $\mathrm{BaCl}_{2}$ 0,2 mol. $\mathrm{L}^{-1}$, em um erlenmeyer e foi submetida à titulação com ácido clorídrico a 0,1 mol. $\mathrm{L}^{-1}$.

O cálculo final da taxa respiratória em cada dia de armazenamento foi realizado com base na média das quinze repetições, cujo resultado foi expresso em mg de $\mathrm{CO}_{2} \cdot \mathrm{g}^{-1} \mathrm{de}$ massa fresca dos frutos, para dia de armazenamento.

\section{Delineamento experimental}

O delineamento experimental utilizado foi o inteiramente casualizado, em que foi considerado como tratamento os dias de armazenamento $(0,1,2,3$ e 4), com 5 repetições (bandejas) de aproximadamente $100 \mathrm{~g}$ de frutos.

Foram determinados os coeficientes de correlação de Pearson entre a massa fresca, perda de massa fresca e a taxa respiratória, durante o armazenamento, por meio do software Microsoft Office Excel versão 2007.

\section{RESULTADOS E DISCUSSÃO}

A massa fresca do fruto não apresentou grandes variações durante o período de armazenamento (Figura 4), variando no geral de 3,26 a 3,30 g, variando em torno de $1,2 \%$.

Figura 4. Massa fresca de frutos de juazeiro armazenados sob temperatura ambiente controlada $\left(23 \pm 1{ }^{\circ} \mathrm{C}\right.$ e $55 \pm 2 \%$ UR), Pombal, Paraíba. A barra vertical representa o desvio padrão da média.

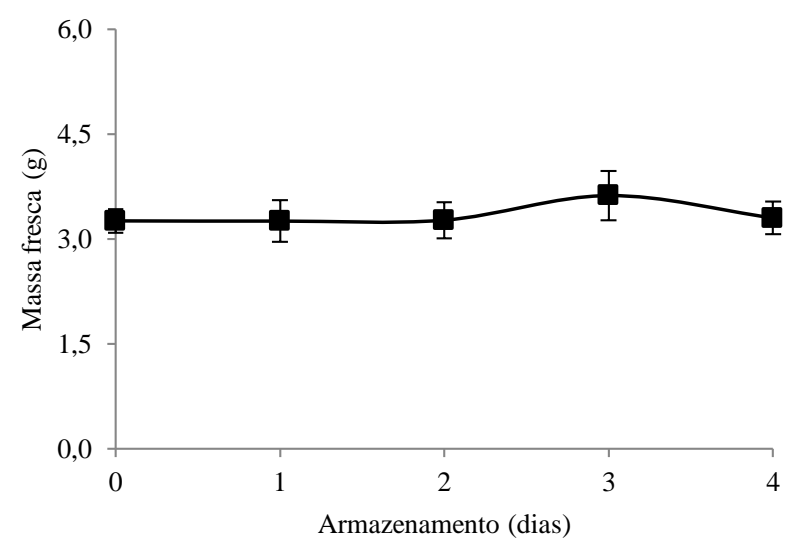

Foi constatado um leve acréscimo no dia 3 de armazenamento seguido por decréscimo no dia 4.

Os valores obtidos nessa pesquisa são próximos aos determinados por Silva et al. (2011), que ao avaliar cinco acessos de juazeiro obteve massa fresca variando de 3,91 a $3,19 \mathrm{~g}$, apesar do valor ser próximo, a variação apresentada 
em porcentagem é superior a determinada nesta pesquisa, sendo em torno de $22,6 \%$. Este fato pode ser atribuído a desuniformidade dos frutos empregados no experimento.

A perda de massa de frutos de juazeiro é retratada na Figura 5.

Figura 5. Perda de massa fresca de frutos de juazeiro armazenados sob temperatura ambiente controlada $\left(23 \pm 1{ }^{\circ} \mathrm{C}\right.$ e $55 \pm 2 \%$ UR). Pombal, Paraíba. A barra vertical representa o desvio padrão da média

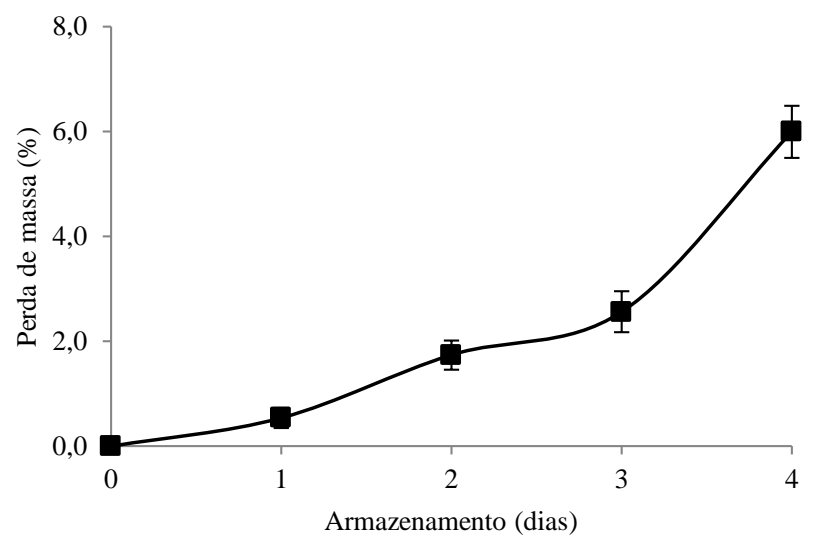

Percebe-se uma elevação na perda de massa durante todo o armazenamento, atingindo cerca de $6 \%$ no último dia de análise. A perda de massa de frutos para Finger e França (2001), é resultado da soma de perda de água pela transpiração e perda de matéria seca devido à atividade respiratória.

Valores superiores foram encontrados por Silva (2015), que ao avaliar frutos de juazeiro no IV estádio de desenvolvimento determinou valores de perda de massa de quase $10 \%$ no $4^{\circ}$ dia de armazenamento a temperatura ambiente. Essa diferença nos resultados pode ser atribuída à diferença nas temperaturas utilizadas, para esse experimento foi empregada uma temperatura de $23 \pm 1{ }^{\circ} \mathrm{C}$, já na pesquisa de Silva (2015) a temperatura variou de $28 \pm 2{ }^{\circ} \mathrm{C}$, o que apresenta grande influência na perda de massa, visto que, quanto maior a temperatura maior será as perdas de água por transpiração e maior a taxa respiratória, resultando em maiores perdas de massa.

A Figura 6 ilustra a taxa respiratória de frutos de juazeiro ao longo do armazenamento. Nota-se elevação da taxa respiratória até o $3^{\circ}$ dia de armazenamento, seguida por decréscimo no último dia de análise. Esse comportamento observado demonstra a ocorrência de pico climatérico respiratório em frutos de juazeiro no $3^{\circ}$ dia de armazenamento.

Para Rhodes (1970) o pico climatérico pode ser definido como um período no qual uma série de mudanças bioquímicas é iniciada por produção autocatalítica de etileno, marcando a transição entre o desenvolvimento e a senescência, envolvendo aumento na respiração e condução ao amadurecimento.

A elevação na taxa respiratória durante o armazenamento pode estar relacionada ao amadurecimento dos frutos durante o armazenamento, a redução na respiração sugere o início da senescência do fruto.

Figura 6. Taxa respiratória de frutos de juazeiro armazenados sob temperatura ambiente controlada $\left(23 \pm 1^{\circ} \mathrm{C}\right.$ e $55 \pm 2 \%$ UR).
Pombal, Paraíba. A barra vertical representa o desvio padrão da média

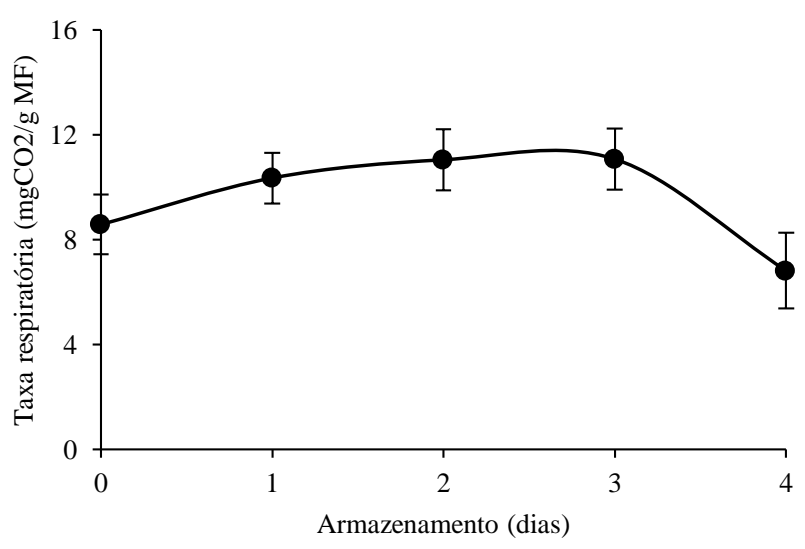

Os valores referentes aos coeficientes da correlação de Pearson para massa fresca, perda de massa e taxa respiratória de frutos de juazeiro são descritos na Tabela 1.

Tabela 1. Coeficientes de correlação de Pearson (r) entre a massa fresca, perda de massa e taxa respiratória de frutos de juazeiro armazenados sob temperatura ambiente controlada $\left(23 \pm 1{ }^{\circ} \mathrm{C}\right.$ e $55 \pm 2 \%$ UR). PPGSA/CCTA/UFCG, Pombal, Paraíba

\begin{tabular}{cccc}
\hline Características & $\begin{array}{c}\text { Massa } \\
\text { fresca }\end{array}$ & $\begin{array}{c}\text { Perda } \\
\text { de massa }\end{array}$ & $\begin{array}{c}\text { Taxa } \\
\text { respiratória }\end{array}$ \\
\hline Massa fresca & 1,000 & & \\
Perda de massa & 0,205 & 1,000 & \\
Taxa respiratória & 0,378 & $-0,556$ & 1,000 \\
\hline
\end{tabular}

Foi determinada leve correlação negativa entre a perda de massa fresca e a taxa respiratória de frutos de juazeiro, onde foi obtido valor de -0,556.

Essa relação aponta comportamento inverso da perda de massa fresca com a taxa respiratória, sendo que à medida que a perda de massa aumenta, a taxa respiratória tende a diminuir.

Fato observado especialmente no último dia de armazenamento (dia 4), onde foi constatado acentuado aumento de perda de massa fresca, migrando de $2,56 \%$ no dia 3 para $5,99 \%$ no dia 4 ; bem como foi verificado redução na taxa respiratória, de $11,06 \mathrm{mg} / \mathrm{CO}_{2} \cdot 100 \mathrm{~g}^{-1} \mathrm{MF}$ no dia 3 a 6,81 $\mathrm{mg} / \mathrm{CO}_{2} / 100 \mathrm{~g}$ MF no dia 4.

Os demais parâmetros avaliados não apresentaram correlação significativa entre si.

\section{CONCLUSÕES}

Os frutos de juazeiro armazenados a temperatura ambiente controlada apresentaram pico respiratório característico de frutos climatéricos, portanto, podem ser denominados como frutos de natureza climatérica.

\section{REFERÊNCIAS}

BRAGA, R. C. JUAZEIRO (Ziziphus Joazeiro) - Árvore da Caatinga com Potencial Medicinal. 2010. Disponível em: http://pro.casa.abril.com.br/group/cronicasdoouroverde/forum /topics/juazeiro-ziziphus-joazeiro-1?xg_source=activity. Acesso em: 15/03/2017. 
CALBO, A. G.; MORETTI, C. L.; HENZ, G. P. Respiração de Frutas e Hortaliças. Brasília, DF, Embrapa, 2007. 10p. (Comunicado Técnico, 46).

CARVALHO, P. M. R. Juazeiro Ziziphus joazeiro. Colombo, PR, Embrapa, 2007. 8p. (Comunicado Técnico, 139).

CRISPIM, J. E.; MARTINS, J. C.; PIRES, J. C.; ROSELEM, C. A.; CAVARIANI, C. Determinação da taxa de respiração em sementes de soja pelo método da titulação. Pesquisa Agropecuária Brasileira, v. 29, n. 10, p.1517-1521, 1994.

DANTAS, F. C. P.; TAVARES, M. L. R.; TARGINO, M. S.; COSTA, A. P.; DANTAS, F. O. Ziziphus joazeiro Mart. Rhamnaceae: características biogeoquímicas e importância no bioma Caatinga. Revista Principia, João Pessoa, n.25, p.52$57,2014$.

FINGER, F. L.; FRANÇA, C. F. M. Pré-resfriamento e conservação de hortaliças folhosas. In: CONGRESSO BRASILEIRO DE OLERICULTURA, 51. Horticultura Brasileira, v. 29, n.2, Viçosa, 2011.

FONSECA, S. C.; OLIVEIRA, F. A. R.; BRECHT, J. K. Modelling respiration rate of fresh fruits and vegetables for modified atmosphere packages: a review. Journal of Food Engineering, Davis, v.52, n.2, p.99-119, 2002.

LOPPES, A. H. Índice terapêutico fitoterápico. Petrópolis: EPUB, 2008.

LORENZI, H. E. Árvores brasileiras: manual de identificação e cultivo de plantas arbóreas nativas do Brasil. 5. ed., v. 1. Nova Odessa, Instituto Plantarum, 2009.

LORENZI, H.; MATOS, F. J. A. Plantas medicinais no Brasil: nativas e exóticas. 2 ed. Nova Odessa, Instituto Plantarum, 2008.

RHODES, M. J. C. The climateric and ripening of fruits. In: HULME, A. C. The Biochemistry of fruits and their products. London: Academic Press, v. 1, p.521-533, 1970.

SILVA, J. L. Qualidade e armazenamento de frutos de juazeiro (Ziziphus joazeiro Mart.) sob temperatura ambiente. Pombal: Universidade Federal de Campina Grande, 2015. 75p. Monografia do curso Bacharel em Engenharia de Alimentos.

SILVA, L. R.; BARRETO, D. S.; BATISTA, P. F.; ARAÚJO, F. A. R.; MORAIS, P. L. D.. Caracterização de frutos de cinco acessos de juazeiro (Ziziphus joazeiro Mart.). Revista Brasileira de Produtos Agroindustriais. Campina Grande, v.13, n.1, p.15-20, 2011. 